\title{
Preface to the special issue on personalization and behavior change
}

\author{
Judith Masthoff · Floriana Grasso · Jaap Ham
}

(C) Springer Science+Business Media Dordrecht 2014

\section{Personalization and behavior change}

Digital behavior intervention is a growing area of research which investigates how interactive systems can encourage and support people to change their behavior. Personalization plays an important role in this, as the most effective persuasive and motivational strategies are likely to depend on user characteristics such as the user's personality, affective state, existing attitudes, behaviors, knowledge, and goals. Example application areas include healthcare (e.g., encouraging people to eat more healthily and exercise more), education (e.g., motivating learners to study more), environment (e.g., encouraging people to use less energy and more public transport), and collaborative content development (e.g., incentivizing people to annotate resources).

The idea that a system may be used to motivate people on the basis of a user model is certainly not novel. As early as the 1980s, intelligent tutoring systems encouraged students to learn by means of tailored feedback and hints (Sleeman and Brown 1982), and in the 90 s patient education systems attempted to address compliance to a medical regimen by means of information and personalized advice (De Carolis et al. 1996) or encouraged people to adopt healthier lifestyles (Reiter et al. 2003). However, it is only recently that a number of seemingly non correlated, extensive research efforts,

\footnotetext{
J. Masthoff $(\bowtie)$

University of Aberdeen, Aberdeen, UK

e-mail: j.masthoff@abdn.ac.uk

F. Grasso

University of Liverpool, Liverpool, UK

e-mail: floriana@liverpool.ac.uk

J. Ham

Eindhoven University of Technology, Eindhoven, The Netherlands

e-mail: J.R.C.Ham@tue.nl
} 
from various perspectives, have started to focus on a more complex cognitive model of rational and extra-rational features involving emotions, persuasion, motivation and argumentation. We can distinguish three parallel strands of research that have become prominent.

The first, stemming from a seminal work by Fogg (2003), is now widely known as Persuasive Technology. Persuasive Technology focuses on interactive technology that can motivate and influence the user. This community of research is now well established, with an International Persuasive conference and smaller events associated with other conferences.

The second research field is Affective Computing. Originated by a seminal work by Picard (1997), Affective Computing is interested in the use, understanding and modelling of emotions and affect in computer systems. Started in the early 1990s, this is now an established discipline, with an international conference (ACII), a professional society (Association for the Advancement of Affective Computing, formerly HUMAINE) and a journal (IEEE Transactions on Affective Computing).

Finally, Argument and Computation (Simari et al. 2011) has emerged in the past decade as a research strand interested in computational models and theories of argumentation and persuasion coming from Philosophy and Artificial Intelligence. Many events are dedicated to the topic, including two annual workshop series (Argumentation in Multi-Agent Systems and Computational Models of Natural Argument), and a biennial international conference (COMMA). These have recently been complemented by a new journal (Argument and Computation).

This special issue connects these three areas of research. It presents analyses and empirical research that focus on how adaptive and personalized systems can motivate people, for instance to improve health (op den Akker et al. 2014; Dijkstra 2014; Orji et al. 2014) and learning (Bouvier et at. 2014).

\section{Theories, techniques and the need for personalization}

Many theories related to behavior change exist, from social psychology, organizational science, behavioral economics, and marketing. Based on five theories, Fishbein et al. (2001) identified 8 determinants of behavior. Michie et al. (2005) extended this to 12 determinants of behavior based on 33 theories, whilst Fogg (2009) only distinguished three core determinants: Ability, Motivation and Trigger. Michie et al. (2008) identified 137 behavior change techniques, and mapped these to the behavioural determinants. Behavior change theories predict effective combinations of techniques. For example, control theory advocates goal setting, specifying action plans, self-monitoring, feedback and reviewing goals. In this special issue, op den Akker et al. (2014) and Orji et al. (2014) discuss behavior change techniques and theories.

Considering some popular behavior change techniques, there clearly is a need for personalization within the techniques. There has for example been research on:

- Tailoring feedback to comparative performance (Colineau and Paris 2011) and adapting performance feedback and emotional support to the user's personality (Dennis et al. 2013).

- Adaptive incentive mechanisms (Cheng and Vassileva 2006). 
- Tailoring prompts. Fogg (2009) distinguishes three types of prompts: spark prompts motivate people who lack motivation to act, facilitator prompts make behavior easier for motivated people with low ability, and signal prompts remind motivated people with high ability to act. Masthoff et al. (2013) showed that the content of prompts in the charity domain needs to be personalized to gender, age, religion and country affinity.

- Adaptation of persuasive arguments to user preferences ( Grasso et al. 2000; Carenini and Moore 2006) and user goals as inferred partially from user personality (Mazzotta et al. 2007). For an argument to be effective, it needs to be within the so-called latitude of acceptance; i.e. not too far from the user's current position. There is initial research on modeling a user's position after hearing arguments, based on argument strength, position and receiver involvement (Nguyen et al. 2007). There is also research on source credibility, showing that the source needs to be adapted to the topic, but without evidence yet of it requiring to be adapted to the user (Nguyen and Masthoff 2008).

- Adaptation to intercultural differences, for example by adapting human-robot greeting behavior to the cultural background of the user (Trovato et al. 2014).

- Increasing similarity between the user and the persuasive technology to improve the effectiveness of trust-related cues: e.g., by increasing appearance (facial) similarity (Verberne et al. 2013), or emphasizing overlap in goals between user and system (Verberne et al. 2012).

- Fitting the extent to which a persuasive strategy needs cognitive processing to the cognitive processing capacity a user has available (Maan et al. 2011).

In addition to personalization within persuasion techniques, there is a need for personalization of the choice of techniques. Several lines of research investigated this mechanism. For example, Schultz et al. (2007) showed that the effectiveness of a persuasion technique based on (descriptive) social norms (detailing average neighborhood usage) for influencing household energy consumption depended on whether a household itself consumed at a high or low rate. Even a user's age might be relevant, as Brown et al. (2005) suggested that older people are more sensitive to Cialdini's (1984) consistency technique. More generally, a series of studies by Kaptein (e.g. Kaptein et al. 2012) has investigated the effectiveness of adapting the strategy used in a persuasive message (for example, appeal to authority, appeal to commitment) to the user's susceptibility to Cialdini's six social persuasion principles. Within this issue, Orji et al. (2014) investigate the adaptation of strategy choice to gamer types.

\section{Papers in this issue}

The collection of papers in this special issue testifies to the broad range of issues that researchers are addressing, and hopefully, with their extensive reference collection, will provide a wide range of perspectives to those who wish to approach this field.

The paper "Tailoring real-time physical activity coaching systems: a literature survey and model" (op den Akker et al. 2014) by Harm op den Akker, Valerie Jones and Hermie Hermens underlines the need to increase adoption of tailoring methods that are based on available theories. It bases this conclusion on a survey of state of the art 
physical activity coaching systems, and presents a conceptual framework which identifies seven important tailoring concepts that are currently in use and how they relate to each other. This contribution shows the necessity of developing innovative evaluation methods to demonstrate the effectiveness of individual tailoring approaches.

The paper "The persuasive effects of personalization through name mentioning in a smoking cessation message" (Dijkstra 2014) by Arie Dijkstra presents evidence that a very basic form of personalization (mentioning the persuadee's name in the persuasive message) needs a strong fit on a relevant but different user characteristic to be effective. That is, this research presents empirical evidence that the effects of mentioning the recipient's name depend on individual differences in personal relevance of the message (health value) before exposure to the message: name mentioning can increase persuasion, but also decrease persuasion through defensive processes.

The paper "A trace-based approach to identifying users' engagement and qualifying their engaged-behaviors in interactive systems: application to a social game" (Bouvier et at. 2014) by Patrice Bouvier, Karim Sehaba and Élise Lavoué describes a model for detecting and classifying players' engagement in digital games. In contrast with many works based on metrics, by combining insights from three theoretical frameworks (Self-Determination Theory, Activity Theory and Trace Theory), it provides a qualitative account of engaging behavior. A user study in a Social Gaming environment, monitored for four months, corroborates the results.

The paper "Modeling the efficacy of persuasive strategies for different gamer types in serious games for health" (Orji et al. 2014) by Rita Orji, Julita Vassileva and Regan Mandryk shows that the choice of behavior change techniques needs to be tailored to the user, in their case to the user's BrainHex gamer type. It provides evidence that strategies that work for certain gamer types may be counterproductive for other gamer types. Finally, it offers a data-driven approach for designing persuasive interventions that are tailored to personality types.

\section{References}

Bouvier, P., Sehaba, K., Lavoué, É.: A trace-based approach to identifying users' engagement and qualifying their engaged-behaviours in interactive systems: application to a social game. User Modeling User-Adapt. Interact. (2014). doi:10.1007/s11257-014-9150-2

Brown, S.L., Asher, T., Cialdini, R.B.: Evidence of a positive relationship between age and preference for consistency. J. Res. Personal. 39, 517-533 (2005)

Carenini, G., Moore, J.D.: Generating and evaluating evaluative arguments. Artif. Intell. 170, 925-952 (2006)

Cheng, R., Vassileva, J.: Design and evaluation of an adaptive incentive mechanism for sustained educational online communities. User Modeling User-Adapt. Interact. 16, 321-348 (2006)

Cialdini, R.B.: Influence: the psychology of persuasion. William Morrow and Company, New York (1984)

Colineau, N., Paris, C.: Motivating reflection about health within the family: the use of goal setting and tailored feedback. User Modeling User-Adapt. Interact. 21, 341-376 (2011). doi:10.1007/ s11257-010-9089-X

De Carolis, B., de Rosis, F., Grasso, F., Rossiello, A., Berry, D.C., Gillie, T.: Generating recipient-centered explanations about drug prescription. Artif. Intell. Med. 8(2), 123-145 (1996)

Dennis, M., Masthoff, J., Mellish C. Does learner conscientiousness matter when generating emotional support in feedback? ACII, 209-214 (2013) doi:10.1109/ACII.2013.41

Dijkstra, A.: The persuasive effects of personalization through name mentioning in a smoking cessation message. User Modeling and User-Adapt. Interact. (2014). doi:10.1007/s11257-014-9147-x 
Fishbein, M., Triandis, H.C., Kanfer, F.H., Becker, M., Middlestadt, S.E., Eichler, A.: Factors influencing behaviour and behaviour change. In: Baum, A., Revenson, T.A., Singer, J.E. (eds.) Handbook of health psychology, pp. 3-17. Lawrence Erlbaum Associates, Mahwah (2001)

Fogg, B.J. Persuasive technology: using computers to change what we think and Do. Morgan Kaufmann. (2003)

Fogg, B.J.: A behavior model for persuasive design. In: 4th international conference on persuasive technology, Claremont, CA, 26-29 (2009)

Grasso, F., Cawsey, A., Jones, R.: Dialectical argumentation to solve conflicts in advice giving: a case study in the promotion of healthy nutrition. Int. J. Human Comput. Studies 53, 1077-1115 (2000). doi:10. 1006/ijhc.2000.0429

Kaptein, M., de Ruyter, B., Markopoulos, P., Aarts, E.: Adaptive persuasive systems: a study of tailored persuasive text messages to reduce snacking. ACM Trans. Interact. Intell. Syst. 2, (2012). doi:10.1145/ 2209310.2209313

Maan, S., Merkus, B., Ham, J., Midden, C.: Making it not too obvious: the effect of ambient light feedback on space heating energy consumption. Energy Effic. 4, 175-183 (2011)

Masthoff, J., Langrial, S., van Deemter, K.: Personalizing triggers for charity actions. Persuasive, 125-136. (2013)

Mazzotta, I., de Rosis, F., Carofiglio, V.: Portia: a user-adapt persuasion system in the healthy-eating domain. IEEE Intell. Syst. 22, 42-51 (2007)

Michie, S., Johnston, M., Abraham, C., Lawton, R., Parker, D., Walker, A.: Making psychological theory useful for implementing evidence based practice: a consensus approach. Qual. Safety Health Care 14, 26-33 (2005)

Michie, S., Johnston, M., Francis, J., Hardeman, W., Eccles, M.: From theory to intervention: mapping theoretically derived behavioural determinants to behaviour change techniques. Appl. Psychol. 57(4), 660-680 (2008)

Nguyen, H., Masthoff, J., Edwards, P.: Modelling a receiver's position to persuasive arguments. Persuasive, 271-282 (2007)

Nguyen, H., Masthoff, J.: Using digital images to enhance the credibility of information. Persuasive technology symposium. (2008)

op den Akker, H., Jones, V.M., Hermens, H.J.: Tailoring real-time physical activity coaching systems: a literature survey and model. User Modeling User-Adapt. Interact. (2014). doi:10.1007/s11257-014-9146-y

Orji, R., Vassileva, J., Mandryk, R.: Modeling the efficacy of persuasive strategies for different gamer types in serious games for health. User Modeling User-Adapt. Interact. (2014). doi:10.1007/s11257-014-9149-8

Picard, R.W.: Affective computing. MIT Press, Cambridge (1997)

Reiter, E., Robertson, R., Osman, L.M.: Lessons from a failure: generating tailored smoking cessation letters. Artif. Intell. 144(1), 41-58 (2003)

Schultz, P.W., Nolan, J.M., Cialdini, R.B., Goldstein, N.J., Griskevicius, V.: The constructive, destructive, and reconstructive power of social norms. Psychol. Sci. 18, 429-434 (2007)

Simari, G.R., Reed, C., Rahwan, I., Grasso, F.: Introducing argument and computation. Argument Comput. 1(1), 1-5 (2011)

Sleeman, D., Brown, J.S.: Intelligent tutoring systems. Academic Press, New York (1982)

Trovato, G., Zecca, M., Sessa, S., Jamone, L., Ham, J., Hashimoto, K., Takanishi, A.: Cross-cultural study on human-robot greeting interaction: acceptance and discomfort by Egyptians and Japanese. Paladyn. J. Behav. Robot. 4, 83-93 (2014)

Verberne, F., Ham, J., Midden, C.: The car that looks like me: similarity cues can increase trust in the self-driving cars of the future. ERCIM News 94, 23-24 (2013)

Verberne, F.M.F., Ham, J., Midden, C.: Trust in smart systems: the influence of sharing user goals and information on trust in and acceptance of smart systems in cars. Human Factors 54, 799-810 (2012). ISI Journal.

Judith Masthoff is a professor and chair in Computing Science at the University of Aberdeen. Her research is in personalisation and intelligent user interfaces. She is interested in personalizing behavior change mechanisms for encouraging people to live more healthily and sustainably, and in adapting motivating and emotional support messages to personality. She has co-organized four workshops on behavior change technology and co-presented a tutorial on personalization for behaviour change at UMAP 2014. She serves on the editorial board of User Modeling and User-Adapted Interaction. She was program chair 
of the UMAP conference in 2012 and is a director of User Modeling Inc., the professional association of user modeling researchers. She has served on the PC of the Persuasive conferences since 2006.

Floriana Grasso is a lecturer in the Department of Computer Science at the University of Liverpool. Her main research focus is computational argumentation, and persuasion technologies, and she applied this to digital interventions to motivate users to adopt a particular behaviour or lifestyle. She sits on the steering committee of the ICST International Conference on eHealth, and she is co-editor in chief of the Argument and Computation journal. Recent work include research on recommendation system technology for patient monitoring, adaptive web technology for health consumer education, and on monitoring and encouraging child language acquisition, part of the newly funded interdisciplinary Centre for Language and Communicative Development.

Jaap Ham is associate professor at Eindhoven University of Technology, The Netherlands. His research focus is on the social psychology of human-technology interaction. He studies questions concerning persuasive technology, user experiences, usability, persuasive robotics, and ambient (persuasive) intelligence, often in close collaboration with industry. He is associate editor of the International Journal on Social Robotics, and published in a variety of international scientific journals. Also, he (co-) organized various scientific conferences (e.g., as chair of the International Conference on Social Robotics, 2011), and conferences and workshops on persuasive technology (e.g., at the annual conference on Ambient Intelligence, and UMAP). 